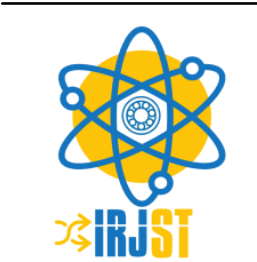

Available online at https://www.irjst.com/

International Research Journal of Science and Technology

ISSN:2707-3955

DOI: https://doi.org/10.46378/irjst.2019.010109

\title{
Examination of Different Inks Available in Ethiopia Using VSC 8000, U.V-Visible Spectrophotometer and TLC: Study of Their Discrimination Potential
}

\section{Natinael Mekonnen*}

Department of Forensic Chemistry and Toxicology, Arba Minch University, Ethiopia.

Published : 2019

\section{Key Words}

Documents,

Dyes

Forgery,

Spectral comparison

VSC

\section{Abstract}

Ink analysis is the most challenging and commonly encountered problem faced by a forensic chemist. Ink present on documents such as contracts, cheques, testaments, medical records, to quote just a few, is regularly contested for its authentication. In this paper, we have examined the various inks available in Ethiopia (speciallyArba Minch) using chromatography and spectroscopy methods, and have tried to decode the chemical compositions of these inks emphasizing on coloring agents followed by evaluating the discrimination potential of these techniques. This study is the first kind of detailed examination of inks available in Ethiopia as there is no precedence literature to it. It was found out that the examined ink samples can be divided into five major groups having mainly rhodamine $\mathrm{b}$, rhodamine $6 \mathrm{G}$ and crystal violet as coloring agents. The TLC and UV-Vis techniques although discriminate inks samples to a little better extent than VSC, but VSC should be preferred due to its non-destructive nature.

Copyright (C) 2019:Natinael Mekonnen.This is an open access distribution, and reproduction in any medium, provided Access article distributed under the Creative Commons Attribution License the original work is properly cited License, which permits unrestricted use.

Citation: Natinael Mekonnen.. "Examination of Different Inks Available in Ethiopia Using VSC 8000, U.V-Visible Spectrophotometer and TLC: Study of Their Discrimination Potential", International Research Journal of Science and Technology, 1(1), 56-65, 2019

\section{Introduction}

Analysis of writing ink on questioned documents is often used in establishing whether a document is authentic or fraudulent. It is determined by examining the ink for the following scientific observations:

(a) Whether written entries come from a common source (same ink formula),

(b) What is the relative age of entries of ink on the documents?

This may require identification of the manufacturer and the specific formula of ink.

\footnotetext{
${ }^{*}$ Corresponding author: NatinaelMekonnen

Department of Forensic Chemistry and Toxicology, Arba Minch

University, Arba Minch, Ethiopia.

Email: natinael.mekonnen@amu.edu.et
}

The other purposes of the examination of an ink specimen in forensic science are to identify its source. The inference of the source of an ink specimen can be achieved by the direct comparison of the ink specimen against particular writing instruments (or writings) considered in a case. In some cases, it may be interesting to gather information on the manufacturer of an ink specimen for intelligence purposes. This is achieved by comparing the specimen to an ink library. The interpretation of the results of the comparison of an ink specimen with control samples considered in a case, or from a library, is very much influenced by the availability of ink with similar characteristics in the ink marketplace. In Ethiopia the financial white collars crimes involving documents and hence inks are increasing at a rapid pace and there is an urgent need to examine the inks available, to study the discrimination potential so that best technique can be 
selected and to develop a database for different inks available in Ethiopia. In the proposed project as much inks will be analyzed as per the time frame and the available resources. The main components of modern inks are a) the coloring agent, b) the vehicle (solvents) and c) additives (resins). Their formulations can be composed of a large variety of natural and synthetic products, organic and/or inorganic components. Here we have focused more on the dye components of the ink, so this is going to be detailed in the coming text. The main objective of the study was to analyze various inks available in Ethiopia using VSC 8000, UVVisible and IR spectrophotometer techniques and to evaluate their discrimination potential.

\subsection{Review of status of research and development in the subject}

Numerous analytical methods have been used in the analysis of inks. The analysis of inks is divided into two categories: destructive and non-destructive. In destructive method, portion of the document with the ink has to be removed prior to analysis. Thin layer chromatography (TLC), high performance thin layer chromatography (HPTLC), electrophoresis, high performance liquid chromatography (HPLC), and gas chromatography (GC) are some of the methods that fall in the first category [1-3]. On the other hand, nondestructive method involves the observation of ink on the documents by means of reflectance technique that allows the observation of the inks spectral characteristics without any alteration of the document [4] such as MALDI-TOF, TOF-SIMS, Raman, ATRFTIR etc [5-9]. Fig. 2 show the detail schematic depiction of various analytical methods used for ink analysis based upon their invasiveness. The analytic method has been highlighted as per the color i.e. destructive analysis by red color, while non-destructive by green color and the minimal invasive by yellow color. In Ethiopia there is no precedence literature available for the forensic ink examination and decoding of its main components.

\subsection{Importance of the current project in the context of current status}

Ink examination is the most challenging task for a forensic chemist due to the minimal quantity of ink available for analysis as well as the importance of documents on which it may be present. Since, our department has resourced the VSC 8000, which is one of the most advanced instruments for questioned documents and ink examination, it will be very important to study different inks available in Ethiopia. Inks have been analysed using UV-Visible and IR spectrophotometer so that a better discriminatory technique was selected. This would be very helpful for international forensic community as well as a comparison can be made among the inks available in Ethiopia and other countries.

\section{Materials and Methods}

\subsection{Instruments/Equipments/Chemicals Used:} VSC 8000 from Foster + Freeman ((The VSC®8000 combines sophisticated digital imaging and multiwavelength LED technology with a clear and efficient software interface, to provide a complete solution to the examination of all forms of questioned documents), United Kingdom, U.V-Visible Spectrophotometer from Shimadzu, JapanSilica coated non-fluorescent TLC plates, Quartz cuvettesEthyl acetate, ethanol, distilled water.Ink Samples: Ink samples were purchased from different shops. The details of the studied ink samples as well as the code provided to them are provided in table 1 .

\subsection{Methodology}

The ink samples were collected and directly studied as well as on the documents using VSC 8000. For inks on documents, uniform straight lines were drawn on blank A4 sheets. For removal of ink from documents a sharp blade was used and the inks were dissolved in an appropriate solvent. For VSC 8000, the inks were analyzed in a nondestructive nature. The UV- Visible spectrums of the inks were studied and the components present in them were identified by the precedence literature of the coloring agents present in inks. The standard reference spectrum of the $\mathrm{CV}$ (Crystal violet), Rhodamine B, Rhodamine $6 \mathrm{G}$, and other available dyes was obtained by precedence literature as these dyes were not available in the department.

\subsubsection{Wavelength of the standard (from precedence literature)}

Rhodamine $\mathrm{B}=560 \mathrm{~nm}$

Crystal violet $=570 \mathrm{~nm}$ or $590 \mathrm{~nm}$

Rhodamine $6 \mathrm{G}=530 \mathrm{~nm}$

Eosin yellow $=520 \mathrm{~nm}$.

\subsubsection{Ultraviolet Analysis}

Inks that were used for UV-Vis analysis were dissolved in the suitable solvent, i.e.ethanol (ethanol was used as a blank solvent). First of all a $2 \mathrm{~cm}$ of ink line was scrapped from documents using a sharp blade which was later dissolved in the ethanol. Absorbance 
spectrum were recorded in the wavelength range 200$1000 \mathrm{~nm}$, where the maximum absorbance from each sample were obtained. For comparison the maximum wave length i.e. $\lambda \max$ and relative peak heights of the samples was taken into consideration.

\subsubsection{TLC Analysis}

The separation of ink components is carried out by TLC. Each of ink is dissolved in a suitable solvent i.e. ethanol. The spots of the ink samples which were applied were approximately $0.5-0.8 \mathrm{~mm}$ in diameter. The ink samples were applied 8-10 times employing a thin capillary. The application point of the ink samples were at $1.5 \mathrm{~cm}$ from the bases of the plate. The mobile phase used was ethyl acetate/ethanol/distilled water (50:30:20) The retardation factor, $\mathrm{Rf}$ and color tones of the separated bands were recorded.

\subsubsection{VSC Analysis}

For examination of ink samples using VSC, spectrums were recorded in UV-Visible range and IR range. To minimize the effect of paper, the blank spectra of paper was recorded first. The blank spectra was subtracted automatically from ink + paper spectra by the software preinstalled in VSC. Only the resultant spectrum of ink samples were available for the analysis and interpretation.

\section{Results and Discussion}

\subsection{Ultraviolet-VisibleSpectrophotometer Analysis}

All mentioned inks were examined by UV-Vis spectrophotometer in the wavelength range from 200$800 \mathrm{~nm}$. Figures (2-6) The $\lambda$ max value of all the ink samples is provided in table 2

The table 3 summarizes the results obtained for UVVis analysis. It was observed that all the ink samples were classified into five different groups based upon their UV-Vis spectrum. The group 1 exhibited a maximum in the range of 200-210 $\mathrm{nm}$ (total 7 samples). This range lies in very short UV range and there are too many chances of noise/errors in this range. Moreover, it was found out that these samples were gel based inks and there is possibility that they might contain a pigment. The pigments are generally active in short UV range only. These brands show the absorbance spectra of ballpoint pen, but gel pen inks samples does not give any peak because it is not soluble in ethanol. All ink samples showed maximum absorbance peak in the wavelength range between $200 \mathrm{nmm}$ and $594 \mathrm{~nm}$.

The sample S10 which exhibited a $\lambda \max$ at $531 \mathrm{~nm}$ might contain rhodamine $6 \mathrm{G}$ as coloring agent. The $\lambda \max$ value of this sample matched with the $\lambda \max$ value of rhodamine $6 \mathrm{G}$. The sample S9 which exhibited a $\lambda \max$ value of $557 \mathrm{~nm}$ might contain rhodamine $b$ or a mixture of rhodamine $b+$ rhodamine $6 \mathrm{G}$. The group five which exhibited $\lambda \max$ value in the range of 580-590 nm might contain crystal violet as the coloring agent. The coloring agent present in sample S2 couldn't be ascertained as no precedence literature was found regardin this $\lambda$ max..

\subsection{TLC Analysis}

TLC results are summarized in table 4 .

Since the Rf values are very close in all the samples (approximate 0.9), it indicates that the ink samples are not effectively classified into different groups using TLC. Sample number S10 \& S13 showed a mixture of coloring agents available in them. The spot resolution was the major drawback observed with the TLC, which could be due to Eddy diffusion, longitudinal diffusion etc. We can infer that the TLC didn't come out to be a better discriminatory technique when compared to UV-Vis spectroscopy.

\subsection{VSC8000 Analysis}

From the mentioned pen some of them are examined by VSC 8000 in the wave length range between 400 to 1000. And except the gel ink pen, all others show maximum absorbance peak. But the peak observed is broad due the interference of the paper. the figure is show in fig(7).

\section{Conclusions}

Based on the obtained results, the analysis of ink is of great interest in the field of forensic, particularly in the examination of questioned documents. Forged documents relating to handwriting and signatures often appear in forensic cases. As these documents are usually written with writing pens, it is therefore of interest to discriminate between the studies comprised of analysis of inks utilizing spectroscopic methods. In spectroscopic methods, the inks were analyzed by means of Ultraviolet visible (UV-Vis) and VSC8000 and Thin layer Chromatography (TLC). UV-Vis analysis was successful in discriminating ballpoint pen inks of different brands. VSC8000 is also analysis the ballpoint pen ink successfully. This technique is the 
most important and non-distractive; no need of sample preparation, but the peak which give is broad due to the interference from the paper. As well as which using thin layer chromatography (TLC) methods could be discriminated also depended on the retardation factor, (Rf). Generally from those three technique the $\mathrm{UV}-\mathrm{V}$ is are more discriminating due to giving sharp peak for each ink sample by reading the solution form and also used to separate the different type of ink sample by giving different maximum peak for all listed above on table(1).

The main conclusions drawn from the projects are as follows:

1. Inks studied generally contain Rhoadmine B, Rhodamine $6 \mathrm{G}$ and crystal violet as coloring agent.

2. Ink which did not give peaks in UV-Vis, spots in TLC as well as peaks in VSC may contain pigments in them.

3. VSC being the non-destructive technique is the preferable method for ink examination.

\section{Acknowledgements}

I would like to thank Department of Chemistry, Arba Minch University, Arba Minch, Ethiopia for facilitated the laboratory for working and my advisor Dr. Ali Raza.

\section{References}

1. RendleD. F, Advances in chemistry applied to forensic science, Chem. Soc. Rev. 34 (2005), pp.(1021-1030).

2. Bureau of Alcohol, Tobacco, Firearms and Explosives; United States Department of Justice, U.S. Bomb Data Center, http://www.atf.gov/publications/factsheets/factshe et-us-bomb-data-center.html

3. CantuA. A, Analytical methods for detecting fraudulent documents, Anal. Chem. 63 (1991), pp.(847-854).

4. ClaybournM, AnsellM, Using Raman spectroscopy to solve crime: inks, questioned documents and fraud, Sci. Justice. 40 (2000), pp.(261-271).

5. BrunelleR. L, CrawfordK. R. Advances in the forensic analysis and dating of writing ink. Charles C Thomas Publisher, 2003.

6. NeumannC, MargotP. "New perspectives in the use of ink evidence in forensic science: Part I. development of a quality assurance process for forensic ink analysis by HPTLC, Forensic Sci. Int.185 (2009), pp.(29-37).

7. Hunger, Klaus, ed. Industrial dyes: chemistry, properties, applications. John Wiley \& Sons, 2007.
8. NeumannC, MargotP, Considerations on the ASTM Standards 1789-04 and 1422-05 on the forensic examination of ink, J. Forensic Sci. 55(2010), pp.(1304-1310).

9. MuehlethalerC, GenevieveM, EsseivaP, The application of chemometrics on Infrared and Raman spectra as a tool for the forensic analysis of paints, Forensic Sci. Int. 209 (2011), pp.(173182). 